\title{
Early Neuroblastic and Astrocytic Differentiation Demonstrated Immunohistochemically in a Small Intraocular Medulloepithelioma
}

\author{
Frederick A. Jakobiec a, b, d, e Fouad R. Zakka a, b, d, e Thanos Papakostas ${ }^{a, c-e}$ \\ Shizuo Mukai ${ }^{a}$ c-e \\ ${ }^{a}$ Department of Ophthalmology, ${ }^{b}$ David G. Cogan Laboratory of Ophthalmic Pathology, ${ }^{\text {CRetina Service, }}$ \\ ${ }^{\mathrm{d}}$ Massachusetts Eye and Ear Infirmary, and ${ }^{\mathrm{e}}$ Harvard Medical School, Boston, MA, USA
}

\section{Established Facts}

- The multilaminar intraocular medullary epithelium arises from a more primitive monolayer of premedullary epithelium of the inner layer of the optic cup.

\section{Novel Insights}

- The medullary epithelium was shown to produce GFAP+ fibrous astrocytes and NeuN- neuroblasts.

\section{Keywords}

Ocular medulloepithelioma · Premedullary epithelium . Medullary epithelium · Astrocytes · Neuroblasts · CRX . NeuN $\cdot$ Neurofilaments $\cdot$ Glial fibrillary acidic protein . $\mathrm{S} 100 \cdot \mathrm{CD} 99$

\footnotetext{
Abstract

Purpose: To investigate the source of fibrous astrocytes and neuroblasts in a small ciliary body medulloepithelioma appearing as a leukocoria in a 3-week-old baby girl. Methods: Histopathologic and immunohistochemical studies included Alcian blue, periodic acid-Schiff, and antisera for the detection of S100 protein, CD99, glial fibrillary acidic protein
}

(GFAP), CRX, NeuN, neurofilaments, synaptophysin, desmin, and myogenin. Results: A small, nonteratoid ciliary body medulloepithelioma with collections of Alcian blue+ mucoplysaccharides was present in the enucleated globe. The retinal mass displayed multilaminar dysplastic rosettes that were $\mathrm{CRX}+, \mathrm{NeuN}-$, and synaptophysin-. Intraretinal neurofilaments and scattered NeuN+ neurocytes were also identified. At the base of the retinal mass ribbons and pseudopapillae of $\mathrm{CRX}+, \mathrm{NeuN}$ - medullary epithelium were found. The latter developed from an S100+ and weakly CD99+ monolayer of premedullary epithelium. GFAP+ fibrous astrocytes and NeuN- neuroblasts streamed from the medullary epithelium. Conclusions: A multilaminar medullary epithelium and a precursor monolayer of premedullary epi-

\section{KARGER}

(c) 2017 S. Karger AG, Basel

E-Mail karger@karger.com

www.karger.com/oop
Frederick A. Jakobiec, MD, DSc

David G. Cogan Laboratory of Ophthalmic Pathology, Suite 328

Massachusetts Eye and Ear Infirmary, 243 Charles Street

Boston, MA 02114 (USA)

E-Mail Fred_Jakobiec@meei.harvard.edu 
thelium were both identified. Neuroblasts and fibrous astrocytes were determined to arise separately from the medullary epithelium. The early stage of tumorigenesis afforded by a small tumor provided the opportunity to discover morphologic and immunohistochemical evidence for these differentiations.

(c) 2017 S. Karger AG, Basel

\section{Introduction}

Ocular medulloepithelioma is a childhood tumor that usually develops from the ciliary body epithelium and presents at an older age (average of 5-7 years) and over 10 times more frequently than retinoblastoma [1]. A similar tumor is found in the brain but has different genetic and epigenetic findings [2-5]. For every $100-150$ retinoblastomas 1 ocular medulloepithelioma may occur. Medulloepitheliomas may also arise in the eyes of adults [6, 7]; some of these tumors appear to be generated from the neuroepithelium of the pars plana [7] as well as more commonly from the ciliary processes [2].

The variegated nature of the cellular composition of ocular medulloepithelioma is a subject of ongoing fascination $[1,2]$. The pseudostratified or multilaminar medullary epithelium has been considered, without solid proof, to be the source of the various differentiations observed within ocular medulloepitheliomas. In large lesions the earliest stages of cytologic differentiation are obliterated. Small lesions (the present case is the smallest ever reported), on the other hand, provide a unique opportunity to resolve the issue of the origins of the various cell types. We discovered with close morphologic evaluation and immunohistochemical methods that neuroblasts (precursors of neurocytes/neurons) and fibrous astrocytes can each directly but separately emerge from the medullary epithelium.

Specifically, we found that certain biomarkers identified immunohistochemically were highly revelatory [2, 7]: NeuN for neurorual differentiation, neurofilament protein for neuronal cell bodies and axons, CRX for photoreceptor differentiation, glial fibrillary acidic protein (GFAP) for astrocytes, CD99 for premature precursor neuroepithelium, and S100 for astrocytes and neurons (including their dendrites and axons). It was discovered that astrocytes and neurocytes (neurons) develop separately and not sequentially (that is, not one from the other) out of the medullary epithelium.

Early Differentiation in Ocular

Medulloepithelioma

\section{Report of a Case}

A 3-week-old baby girl was referred to our retinal service with a history of a left leukocoria. Her birth weight was 8 pounds at fullterm with an uncomplicated delivery. There was no family history of an ocular tumor. The infant was able to fix and follow with her right eye. Examination under anesthesia revealed intraocular tensions of $8 \mathrm{~mm} \mathrm{Hg}$ in the right eye and $18 \mathrm{~mm} \mathrm{Hg}$ in the left. Anterior segment examination of the left eye disclosed a central corneal haze and a flat anterior chamber with complete iridocorneal adhesion. Ectropion uveae was observed without iris neovascularization. A dense white plaque was observed in the pupil posterior to the lens (Fig. 1a). The diameter of the left cornea measured 9.5 $\mathrm{mm}$ horizontally and $9.5 \mathrm{~mm}$ vertically $(10 \times 10 \mathrm{~mm}$ on the right). Dilated fundus examination of the right eye demonstrated a normal posterior pole and vasculature. Examination of the left eye showed a white plaque with radial vessels posterior to the lens; the posterior segment could not be visualized. Scleral depression disclosed prominent ciliary processes. Ultrasound biomicroscopy demonstrated obliteration of the anterior chamber and moderately reflective echoes posterior to the iris. B-scan ultrasonography showed diffuse thickening posterior to the lens. A mass $7.5 \mathrm{~mm}$ in elevation emanated from the ciliary body region that displayed moderately reflective interfaces (Fig. 1b). The diagnosis was probable persistent hyperplastic primary vitreous with a possibly associated tumor. It was recommended that the patient have an enucleation given the profound ocular disorganization seen on ultrasonography.

The enucleated globe had a smooth scleral surface and grossly measured $15 \times 18 \times 16 \mathrm{~mm}$. The cornea measured $10 \mathrm{~mm}$ horizontally and $8 \mathrm{~mm}$ vertically. There were $12 \mathrm{~mm}$ of attached optic nerve. Upon sectioning, a $4 \times 3 \mathrm{~mm}$ mass was observed behind the iris and lens. Microscopic evaluation revealed an incompletely cleaved fetal chamber angle and a small tumor arising in the ciliary body and retrolenticular regions (Fig. 1c). The lesion rested against the lens capsule which had been interrupted and partially stripped off the lens cortex (Fig. 1c). The lens had not been invaded by either tumor or fibrous tissue. Elongated ciliary processes touched the equator of the lens. The tumor itself at the base of the mass, near the ciliary body and behind the lens, was composed of fronds, cords, or pseudopapillae (lacking connective tissue cores) (Fig. 1c, d). Most of the mass, however, was constituted by disorganized and immature, compacted retinal tissue in the midst of which was a patent hyaloid artery remnant (Fig. $1 \mathrm{c}$ and inset). The latter extended fibrous bands behind the lens. Elongated astrocytes were observed in a bundle at the apex of the retinal mass. A funnel detachment of the retina was not seen, the result of a tractional disinsertion leaving only a papillary stump at the optic nerve head. The optic nerve was atrophic but not involved by tumor.

Closer examination of the medullary epithelial fronds disclosed pseudostratification of cells with elongated nuclei and wider nuclei in comparison with those of the normal ciliary epithelium (Fig. 1d). Pockets of Alcian blue-positive mucoplysaccharides were found between the medullary strands (Fig. 1d inset). The medullary epithelium originated from an infolding of a monolayer of premedullary epithelium close to the ciliary body and lens (Fig. 1e). At the apex of the fronds was a prominent layer of PAS-positive basement membrane separating the small tumor component from the larger contribution of the overlying dysplastic retina. Small neuroblastic round cells appeared to delaminate from folds of the medullary

Ocul Oncol Pathol 2018;4:176-181 


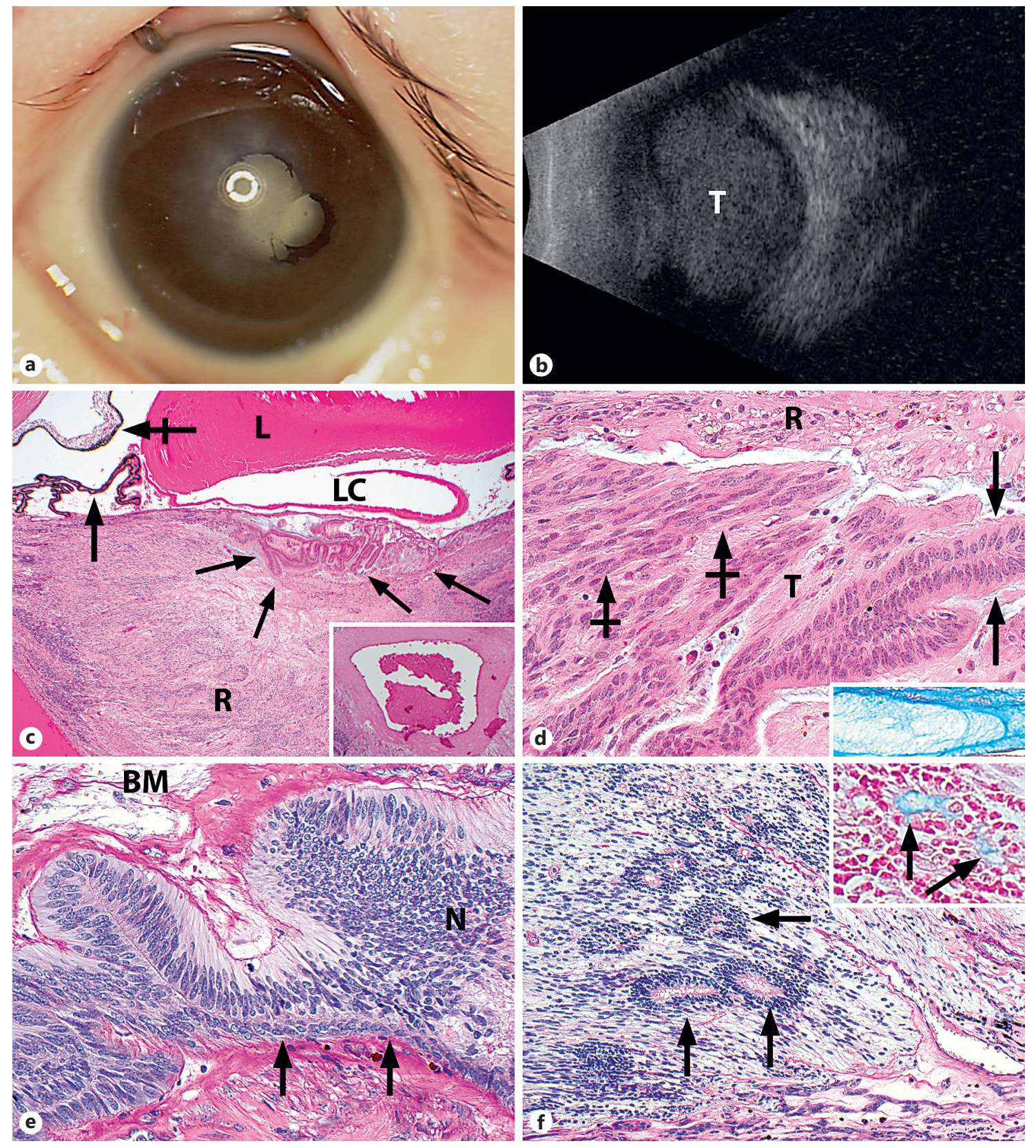

Fig. 1. Small intraocular medulloepithelioma. a A 3-week-old baby girl was noted by her parents to have a left leukocoria. b B-scan ultrasonogram reveals an intraocular tumor $(\mathrm{T})$. A funnel-shaped retinal detachment was not visualized ultrasonographically. c The leukocoria was due to a retrolental (L) mass. The arrows demarcate the component that is a medulloepithelioma with interconnecting cords and ribbons of medullary epithelium. The largest part of the mass was composed of disorganized and compacted retinal tissue (R). LC, ruptured lens capsule. The arrows show elongated ciliary processes; the crossed arrow indicates the iris leaflet. The inset shows a patent persistent hyaloid artery. $\mathbf{d}$ The tumor (T) at the base of the dysplastic retinal/medulloepitheliomatous mass is composed of elongated pseudopapillae and ribbon formations of medullary epithelium. The tumor cells are pseudostratified (arrows). Streaming medullary cells with a less clear-cut structure are seen nearby (crossed arrows). The inset discloses a small pool of mucopolysaccharides. e The monolayer of premedullary epithelium at the base of the tumor (arrows) festoons into the medullary epithelium on the left. Some of the medullary epithelium delaminates into neuroblasts $(\mathrm{N})$. Basement membrane material (BM) separates the tumor elements from the overlying dysplastic retina. $f$ Multilaminar dysplastic rosettes (arrows) are found in the mass of disorganized retinal tissue. The inset highlights the presence of Alcian blue staining mucoplysaccharides in their lumens (arrows). c, d Hematoxylin and eosin. $\mathbf{c} \times 10$. Inset $\times 20$. d $\times 40$. Inset Alcian blue. $\times 20$. e, $\mathbf{f}$ Periodic acid-Schiff. e $\times 40$. $\mathbf{f} \times 20$. Inset Alcian blue. $\times 40$. 

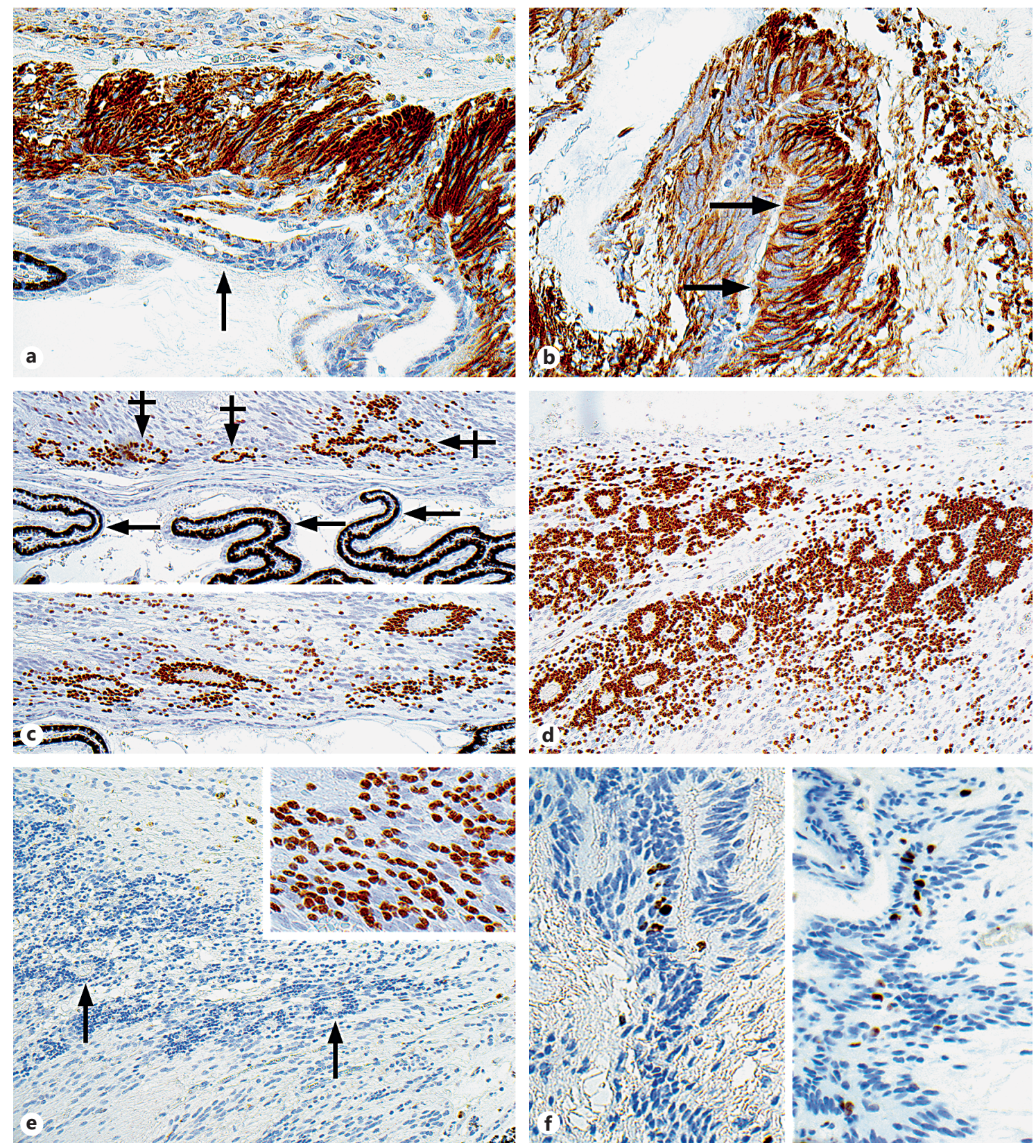

Fig. 2. Immunohistochemical testing results of small intraocular medulloepithelioma. a Prominent glial fibrillary acidic protein staining of a tangle of fibrous astrocytic processes at the level of the medullary epithelium at the base of the mass. Beneath the medullary epithelium is a monolayer of nonstaining premedullary epithelium (arrow). b A medullary frond displays the emergence of fibrous astrocytes (arrows). c Top: CRX positivity is identified in profiles of the medullary fronds (crossed arrows) just above the normal but elongated ciliary processes (uncrossed arrows). Bottom: higher power of CRX+ medullary epithelial formations cut in cross-section. $\mathbf{d}$ CRX is also demonstrable in the dysplastic rosettes of the disorganized retinal mass overlying the neoplasm. e NeuN is not expressed within the dysplastic rosettes (arrows) and surrounding small neuroblasts. The inset reveals that dispersed single neurocytes within the retinal mass are $\mathrm{NeuN}+$. $\mathbf{f} \mathrm{Ki}-67$ protein is limited to the medullary epithelial fronds, where it is located in the nuclei highlighted by dark brown reaction product. The Ki-67 proliferation index was low $(<5 \%)$. a-f Immunoperoxidase reaction, diaminobenzidine chromogen, hematoxylin counterstain. $\mathbf{a} \times 40$, glial fibrillary acid protein. $\mathbf{b} \times 40$, glial fibrillary acid protein. $\mathbf{c} \times 20$ (top) and $\times 40$ (bottom), CRX. d $\times 20$, CRX. e $\times 20$. Inset $\times 40$, NeuN. f $\times 40$ (left and right), Ki67.
Early Differentiation in Ocular Medulloepithelioma
Ocul Oncol Pathol 2018;4:176-181 DOI: 10.1159/000481287 
epithelium (Fig. 1e). Scattered throughout the retinal mass were small round cells, a faint neuropil, and dysplastic rosettes constituted by several layers of cells. The lumens of the rosettes contained Alcian blue staining material (Fig. If and inset).

The premedullary but not the medullary epithelium immunostained strongly for S100 protein and weakly for CD99. Most strikingly, masses of glial cell processes, positive for GFAP (Fig. 2a, b), and NeuN- neuroblasts, arose from the fronds of CRX and S100 medullary epithelium but not from the premedullary epithelium (Fig. 2a). GFAP+ fibrous astrocytes were discovered in the retinal mass mostly along its apical (subretinal) surface. At the tumor's base, near the ciliary body processes and the lens (Fig. 2c, top and bottom), clusters of cells in poorly formed pseudopapillae were CRX+ but NeuN-; CRX positivity was also observed in the rosettes of the dysplastic retina (Fig. 2 d). NeuN positivity was not identified in the retinal dysplastic rosettes (Fig. 2e) but it was limited to several foci of neurocytes in the neuropil of the retinal mass (Fig. 2e, inset). Neurofilaments were detected immunohistochemically in the retinal mass but synaptophysin was negative, implying the absence of synaptogenesis. Desmin and myogenin were negative, ruling out heterologous striated muscle tumor cells (teratoid variant of medulloepithelioma). A low Ki-67 proliferation index $(<5 \%$ of cells) was discovered in the medullary fronds of the tumor (Fig. 2f, left and right) and was $<1 \%$ in the dysplastic retinal mass.

\section{Discussion}

In some medulloepitheliomas, there exists a monolayer referred to as the premedullary epithelium (S100+, CRX-) [2]. The latter can be seen at the periphery of the tumor, both at its base and abutting the lens. The premedullary epithelium undergoes a transition into a pseudostratified or multilaminar medullary epithelium $(\mathrm{CRX}+)$ with fronds, ribbons, and pseudopapillary and cyst formations - punctuated by pools of mucpolysaccharides $[1,2,8]$. In only one published lesion has there been a microscopic suggestion of astrocytic [Fig. 6, top left and top right; 2] and neurocytic [Fig. 5, top left and top right; 2] differentiation focally emanating from the medullary epithelium. In the current small tumor, immunohistochemistry confirmed diffuse astrocytic $(\mathrm{GFAP}+)$ and small-cell neuroblastic (NeuN-) differentiation intimately related to the region of the neoplastic medullary epithelium. CRX is a marker of photoreceptor commitment, while NeuN identifies neuronal differentiation. Astrocytes, neuroblasts, and neurons derive separately (i.e., in parallel, not in sequence) from the medullary epithelium. NeuN+ neurons were scattered in the retinal mass; immunohistochemical identification of associated neurofilaments intimated the presence of axonal processes and also established the existence of neuronal differentiation.
The present tumor was found in a microphthalmic globe with evidence of persistent hyperplastic primary vitreous. This anomaly has been reported in $20 \%$ of medulloepitheliomas, [5] while a persistently patent hyaloid artery also observed in our case can be detected in over $6 \%$ of eyes harboring a medulloepithelioma [1]. The detached retina had not adopted a funnel shape but instead was plastered as a compact mass against the underlying medulloepithelioma and separated from the tumor by redundant, periodic acid-Schiff-positive basement membrane material. The retinal mass was disorganized without lamination and contained dysplastic CRX+ and NeuN- rosettes several cells in thickness [9] that manifested lumenal mucopolysaccharides. The preceding findings are compatible with a tumor developing at an early stage of ocular development. Through traction exerted by the persistent hyperplastic primary vitreous, a disinsertion of the immature retina at the nerve head had occurred, leading to arrested retinal development and permitting only abortive differentiations.

No evidence of heteroplastic or teratoid differentiation $[1,2,5,7,10]$ (striated muscle or cartilage) was found in the present case. Once considered to be a heterologous element, the appearance of a neuropil with neurocytic/ ganglionic differentiation in a medulloepithelioma is probably not a teratoid feature but is within the repertoire of the ocular medullary epithelium itself [2]. The possible origin of striated muscle and cartilage from the medullary epithelium remains moot; the epithelium could conceivably induce these divergent cell types in the tumor's stroma.

\section{Ethics Statement}

This study followed the tenets of the Declaration of Helsinki and is compliant with Health Insurance Portability and Accountability Act's rules and regulations. The Institutional Review Board of the Massachusetts Eye and Ear Infirmary waived its review process. The parents of the patient agreed to the publication of her case.

\section{Disclosure Statement}

The authors have no conflicts to disclose. 


\section{References}

1 Kaliki S, Shields CL, Eagle RC Jr, Vemuganti GK, Almeida A, Manjandavida FP, Mulay K, Honavar SG, Shields JA: Ciliary body medulloepithelioma: analysis of 41 cases. Ophthalmology 2013;120:2552-2559.

2 Jakobiec FA, Trief D, Rashid A, Rose MF, Minckler D, Vanderveen D, Mukai S: New insights into the development of infantile intraocular medulloepithelioma. Am J Ophthalmol 2014;158:1275-1296.

3 Jakobiec FA, Kool M, Stagner AM, Pfister SM, Eagle RC, Proia AD, Korshunov A: Intraocular medulloepitheliomas and embryonal tumors with multilayered rosettes of the brain: comparative roles of LIN28A and C19MC. Am J Ophthalmol 2015;159:10651074.e1.
4 Korshunov A, Jakobiec FA, Eberhart CG, Hovestadt V, Capper D, Jones DT, Sturm D, Stagner AM, Edward DP, Eagle RC, Proia AD, Koch A, Ryzhova M, Ektova A, Schüller U, Zheludkova O, Lichter P, von Deimling A, Pfister SM, Kool M: Comparative integrated molecular analysis of intraocular medulloepitheliomas and central nervous system embryonal tumors with multilayered rosettes confirms that they are distinct nosologic entities. Neuropathology 2015;35:538-544.

5 Sahm F, Jakobiec FA, Meyer J, Schrimpf D, Eberhart CG, Hovestadt V, Capper D, Lambo S, Ryzhova M, Schüller U, Zheludkova O, Kumirova E, Lichter P, von Deimling A, Jones DT, Pfister SM, Kool M, Korshunov A: Somatic mutations of DICER1 and KMT2D are frequent in intraocular medulloepitheliomas. Genes Chromosomes Cancer 2016;55:418427.

6 Ali MJ, Honavar SG, Vemuganti GK: Ciliary body medulloepithelioma in an adult. Surv Ophthalmol 2013;58:266-272.
7 Jakobiec FA, Rose MF, Trief D, Stagner AM, Kim I, Gragoudas ES: Immunohistochemical investigations of adult intraocular medulloepitheliomas. Clin Exp Ophthalmol 2015;43: 379-385.

8 Broughton WL, Zimmerman LE: A clinicopathologic study of 56 cases of intraocular medulloepitheliomas. Am J Ophthalmol 1978;85:407-418.

9 Jakobiec FA, Zakka FR, D'Amato R, Deangelis MM, Walton DS, Rao RC: Unilateral sporadic retinal dysplasia: results of histopathologic, immunohistochemical, chromosomal, genetic, and VEGF-A analyses. J AAPOS 2011;15:579-586.

10 Jakobiec FA, Borkar DS, Stagner AM, Lee NG Intraocular teratoid medulloepithelioma presenting with a completely rhabdomyosarcomatous distant metastasis. JAMA Ophthalmol 2016;134:919-923. 